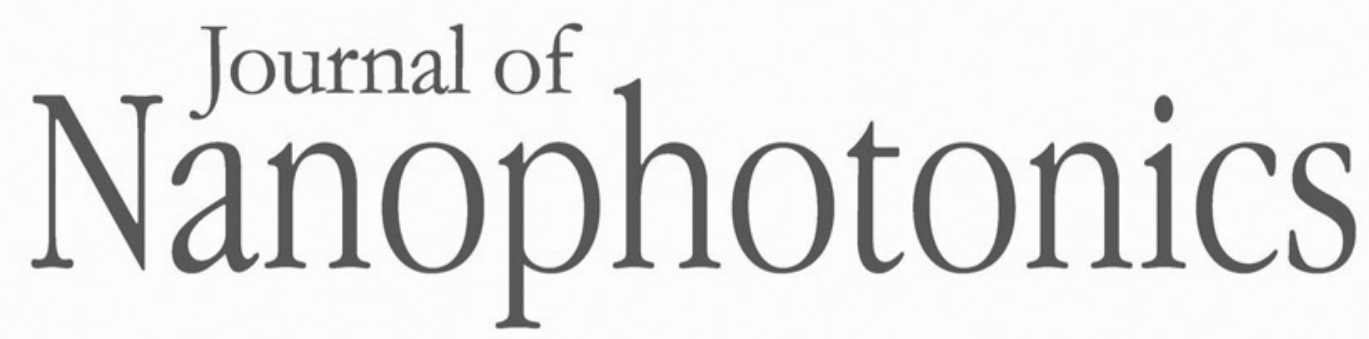

\title{
Modified stripe waveguide design for plasmonic input port structures
}

Evgeniy Panchenko

Timothy D. James

Ann Roberts 


\title{
Modified stripe waveguide design for plasmonic input port structures
}

\author{
Evgeniy Panchenko,* Timothy D. James, and Ann Roberts \\ University of Melbourne, School of Physics, Corner of Swanston Street and Tin Alley, \\ 192 David Caro Building, Melbourne, Victoria 3010, Australia
}

\begin{abstract}
We present a modified asymmetric stripe plasmonic waveguide design for plasmonic integrated input port structures. Such a waveguide shape can significantly increase the surface plasmon polariton (SPP) propagation length. A computational investigation of the waveguide mode analysis, excitation, and guiding is presented as well as SPP propagation length improvement strategies. The proposed structure has the potential to be CMOS compatible and could be used in highly integrated optoelectric circuits. (C) 2016 Society of Photo-Optical Instrumentation Engineers (SPIE) [DOI: 10.1117/1.JNP.10.016019]
\end{abstract}

Keywords: integrated optics; waveguides; plasmons; nanostructures.

Paper 16009 received Jan. 20, 2016; accepted for publication Mar. 8, 2016; published online Mar. 29, 2016.

\section{Introduction}

The speed of integrated electronic circuits is gradually approaching its theoretical limits. These fundamental limitations are determined by interconnections between different parts of the electronic circuit, ${ }^{1}$ the increase in the power dissipation associated with more rapid switching of transistors, as well as the physical limits of the propagation velocity of the electrical signal in the chip. One solution to this problem lies in the utilization of recent advances in optical technology.

The idea of using light as the fundamental basis for computing is not new. ${ }^{2,3}$ Photons, by their physical characteristics, are fundamentally different from electrons. The wave nature of light leads to numerous advantages of optical technologies for the transmission, recording, processing, and storage of information. Optical logic elements can potentially exceed the existing electronic devices in speed as well as in noise immunity. ${ }^{4,5}$ This technology allows the creation of not only optical components for high-speed data transmission via fiber optic, but also all-optical processors that perform high-speed computing with no intermediate signal conversion from optical to electrical.

Unfortunately, devices based on the principles of photonics also have their own drawbacks. The most significant of these is the fact that photonic devices are much larger in size compared with classical electronic devices. That disadvantage is due to the wave nature of light itself, namely, the effect of diffraction. ${ }^{6}$ This fact significantly limits the capacity to integrate such photonic logic elements into complex chips such as microcontrollers or processors.

A more promising technology, in terms of very large-scale integration (VLSI), is the emerging field of plasmonics. It utilizes surface plasmons, which are oscillations of the electron gas at the interface between metal and dielectric. Passive devices, such as wave plates, ${ }^{7}$ filters, ${ }^{8}$ and logic gates, ${ }^{9}$ as well as active elements, such as tunable antennas ${ }^{10-12}$ and detectors ${ }^{13}$ utilizing plasmonic phenomena, have been demonstrated. All these devices exhibit subwavelength confinement of the light and strong electric field enhancement.

Surface plasmons can exhibit very strong field localization, and their wavelength can also be much less than the wavelength of the excitation light, leading to device dimensions significantly less than that of corresponding bulk optical components.

*Address all correspondence to: Evgeniy Panchenko, E-mail: epanchenko@student.unimelb.edu.au

1934-2608/2016/\$25.00 (C) 2016 SPIE 
Due to the mismatch in the wave vectors between surface plasmon polaritons (SPPs) and freely propagating light, SPPs cannot be excited by direct irradiation of a metal surface with light. Several techniques are commonly used to match the vectors, i.e., prism coupling, ${ }^{14,15}$ grating coupling, ${ }^{16}$ and near-field ${ }^{17}$ excitation. Considerable research has been previously undertaken into different techniques of coupling light into SPPs. ${ }^{18}$

A need remains, however, for improved designs to compensate for the high level of loss in plasmonic waveguides. ${ }^{19}$ In this work, we present a flexible design for a waveguide with a modified profile, exhibiting lower loss than a conventional plasmonic stripe waveguide. Furthermore, we consider the performance of the waveguide in conjunction with a grating coupler to form a plasmonic input port, which can be embedded in VLSI circuits. This port structure is suitable for far-field excitation of the surface plasmons and does not require unwieldy laboratory equipment. Commercial optoelectronic chips can possibly be created by combining this plasmonic integrated circuit port design and vertical-cavity surface-emitting lasers using flip chip ${ }^{20}$ technology.

\section{Material and Methods}

Finite element method analysis, implemented in COMSOL Multiphysics 4.3a with RF module, was used to simulate the far-field excitation of SPPs (three-dimensional model) as well as to find the modes supported by the waveguide (two-dimensional model). The optical properties of materials used in the model were taken from experimental data: $\epsilon_{\mathrm{Ag}}=-18.295-0.481 i,{ }^{21}$ $\epsilon_{\mathrm{Si}}=15.032-0.007 i,{ }^{22}$ and $\epsilon_{\mathrm{SiO}_{2}}=2.123$. A plane wave with electric field perpendicular to gratings and a strength of $1 \mathrm{~V} / \mathrm{m}$ was used to excite SPPs. The waveguide length of $20 \mu \mathrm{m}$ was used to minimize the influence of reflections from its end since the waveguide length is much larger than the SPP propagation length. A perfectly matched layer was used together with scattering boundary conditions to decrease reflections from model's boundaries. A wavelength of $\lambda_{0}=632.8 \mathrm{~nm}$ was chosen for SPP excitation.

\section{Design}

A suitable input port should be compatible with far-field surface plasmon excitation without additional equipment, be VLSI and CMOS compatible, and have compact dimensions. Also, the use of normally incident illumination is preferable as the port will be easier to access in multilayer integrated circuits.

The whole structure can be divided into three main components: an excitation grating section, a tapered section, and the waveguide. As can be seen from Fig. 1, the port is implemented on a silicon substrate, where silver was chosen as a material for the waveguide. A thin layer of Ti was

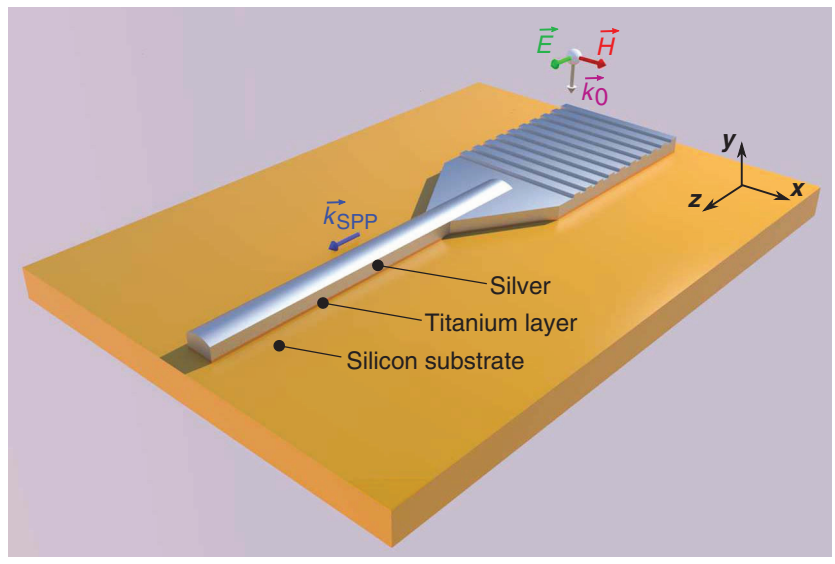

Fig. 1 Schematic representation of plasmonic input port coupled with a modified stripe waveguide. Incident $p$-polarized light with momentum $k_{0}$ excites an SPP (with momentum $k_{\mathrm{SPP}}$ ), which is focused into a waveguide. 
included to improve the typically poor adhesion of Ag to Si. Moreover, the model includes a $2 \mu \mathrm{m}$ passivation layer of silica (not shown in Fig. 1) to prevent oxidation and degradation of silver and also to improve field confinement.

In the proposed structure, SPPs are excited using grating coupling ${ }^{23}$ permitting far-field excitation. A maximum coupling efficiency of $50 \%$ was numerically demonstrated. ${ }^{24}$ The grating structure dimensions are dependent on the materials used, the excitation wavelength, and the angle of illumination (here assumed normal). Since the excitation part of the port was chosen to be much wider than the excitation wavelength and the waveguide is thick enough to decouple SPPs propagating along the upper and lower surfaces of the film, the wave vector of the surface plasmon can be calculated as a two-dimensional metal-dielectric interface. The SPP dispersion curve on an $\mathrm{Ag}-\mathrm{SiO}_{2}$ interface was analyzed to design the grating structure. The optical properties of silver from Ref. 21 were used to define the curve. The SPP propagation length for an infinitely wide interface can be calculated using

$$
L=\frac{1}{2}[\operatorname{Im}(\beta)]^{-1},
$$

where $\beta$ is the propagation constant of the SPP.

As mentioned previously, the structure is designed to work with normally incident illumination, so the SPP momentum is determined only by the grating period $a$.

$$
k_{\mathrm{SPP}}=\frac{2 \pi}{a} .
$$

The SPP wavevector in this case is equal to $k_{\mathrm{SPP}}=15.47 \mu \mathrm{m}^{-1}$, giving a grating period of $a=380 \mathrm{~nm}$. Analysis of a parametric sweep over the grating depth showed that the maximum coupling efficiency from the far-field into surface plasmons is achieved with a grating depth of $200 \mathrm{~nm}$. This can be explained by the fact that Eq. (2) is valid only in the case of shallow gratings ${ }^{25}$ since for deeper ones, the side modes can decrease the coupling efficiency. The waveguide was initially chosen to have a width $w$ of $3 \mu \mathrm{m}$ and thickness $t$ of $60 \mathrm{~nm}$. Such parameters permit improving the far-field coupling and decoupling of top and bottom surface plasmons in a stripe waveguide, reducing attenuation.

Despite the relatively low attenuation (the propagation length is $8.01 \mu \mathrm{m}$ ), the $3-\mu \mathrm{m}$-wide waveguide is too large for VLSI plasmonic circuits. To be suitable for this application, the waveguide was narrowed, leaving the excitation section unchanged. A waveguide width of $s=300 \mathrm{~nm}$ was chosen.
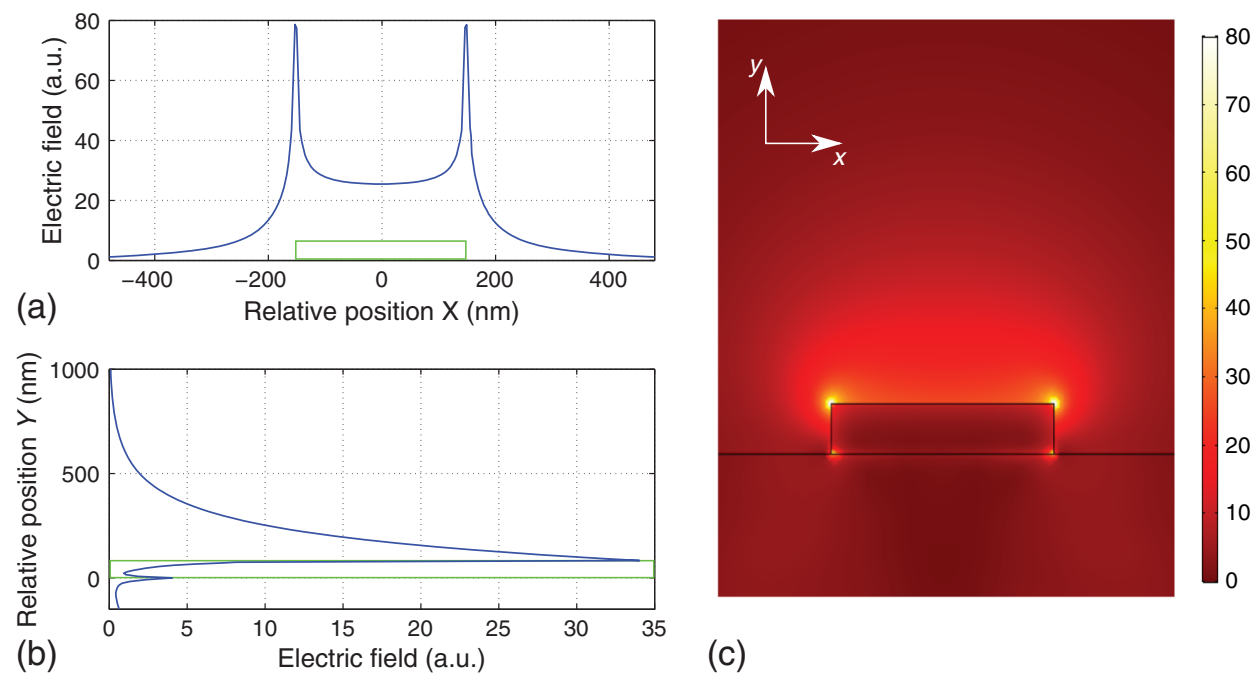

Fig. 2 The normalized electric field distribution along (a) $x$ and (b) $y$ coordinates and (c) normalized electric field profile of rectangular shape waveguide. Green curve represents the waveguide outline. 
To identify the propagating plasmon modes supported by a narrow waveguide with a rectangular cross-section, a numerical study was undertaken. Such a study cannot be performed analytically; ${ }^{26}$ therefore, a numerical approach was used to find supported modes. The fundamental mode $\mathrm{ss}_{b}^{0}$ of the structure should have the smallest imaginary part of propagation constant and hence the lowest attenuation. This mode was found to have a propagation constant of $\beta=$ $15.48-0.432 i \mu \mathrm{m}^{-1}$ and a propagation length of $\sim 1.16 \mu \mathrm{m}$ along the waveguide. The electric field distribution of the fundamental mode is shown in Fig. 2. The distribution shows that in the rectangular stripe waveguide, the energy is mostly guided along the edges. This leads to strong attenuation due to the high field confinement at the corners and thus a short propagation length.

\section{Waveguide Improvement}

It is possible to increase the propagation length by changing the geometry of the waveguide to decrease the field confinement. The strategy used in this case is an elimination of the sharp edges. In the extreme case, the waveguide should not contain any corners. This could be achieved by creating a semicircular profile (see Fig. 3) at the top surface of the waveguide. The electric field profile of such a waveguide is shown in Fig. 4(a).

Introducing a circular profile leads to a uniform distribution of the field along the top surface of the waveguide. The propagation constant in this case is $\beta=15.96-0.101 i \mu \mathrm{m}^{-1}$ and the propagation distance is $4.98 \mu \mathrm{m}$, respectively, which is 4.3 times greater than in the case of rectangular shape.

To understand the fundamental mode evolution, an analysis of the waveguide design for different radii of surface curvature was performed. As an example, Fig. 4(b) represents the field distribution in the case of a curvature of $300 \mathrm{~nm}$. The propagation constant of the waveguide with such a cross-section is $\beta=15.49-0.256 i \mu \mathrm{m}^{-1}$ with propagation length increased by 2.54 times that of the rectangular profile waveguide.

The evolution of the real (blue) and imaginary (green) parts of the effective mode index with radius of curvature as a parameter is shown in Fig. 5. As can be seen, on increasing the radius of curvature, the propagation constant of the mode approaches the value of the rectangular

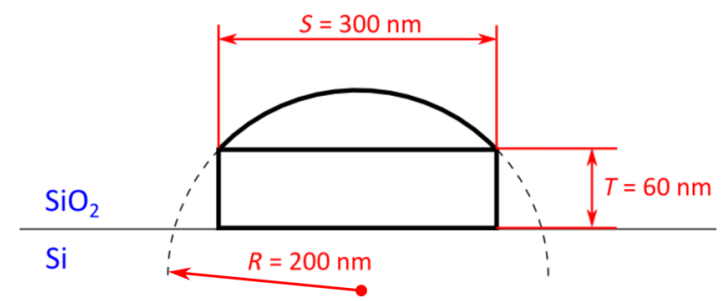

Fig. 3 The cross-section of a modified stripe waveguide. The top surface is curved with the radius of $200 \mathrm{~nm}$ to decrease attenuation.

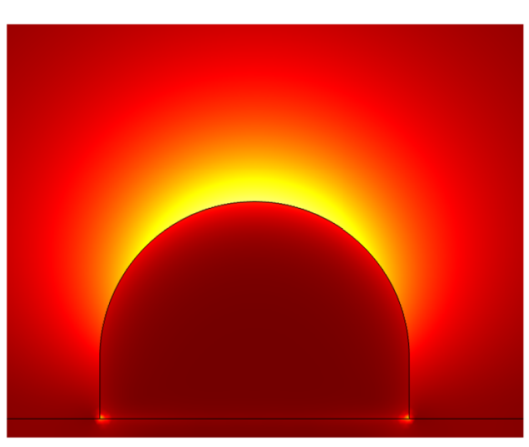

(a)

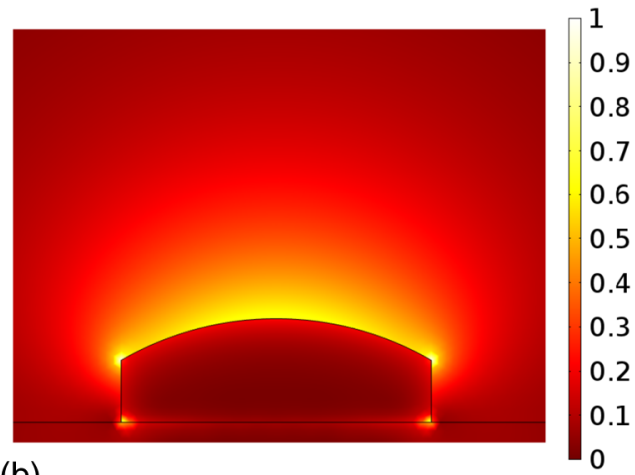

(b)

Fig. 4 The normalized electric field profile of the modified stripe waveguide with circle radius of (a) $150 \mathrm{~nm}$ and (b) $300 \mathrm{~nm}$, respectively. 


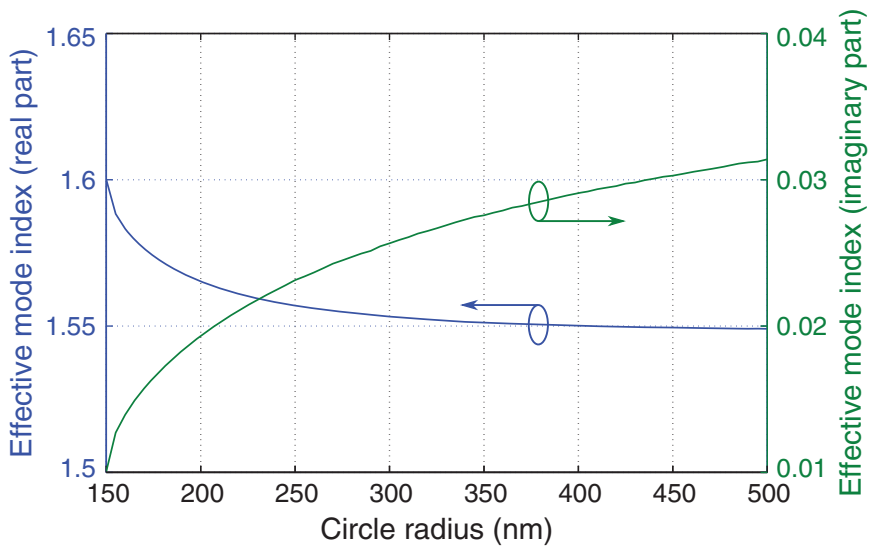

Fig. 5 The evolution of the real (blue) and imaginary (green) parts of the effective mode index. The waveguide mode index approaches that of a rectangular waveguide as the radius of curvature is increased $\left(n_{\text {rec }}=1.55-0.041 i\right)$.

waveguide. Increasing the metal thickness of a waveguide will decrease the leakage into the substrate, and therefore the imaginary part of an effective mode index. However, the improvement in propagation length observed in the hybrid-shaped waveguide over a stripe waveguide cannot be described by just decreasing the mode leakage into the substrate via the increased thickness caused by the waveguides circular profile. In our simulations, we observed up to a $14 \%$ decrease in the imaginary part of the effective index for a given thickness for the hybrid-shape waveguide compared to the stripe waveguide, which results in similar increase of SPP propagation length.

The normalized electric field distribution ( $2 \mathrm{~nm}$ above the surface) along the initial and the fully curved waveguides is shown in Fig. 6(a). The z-components of the field are presented in
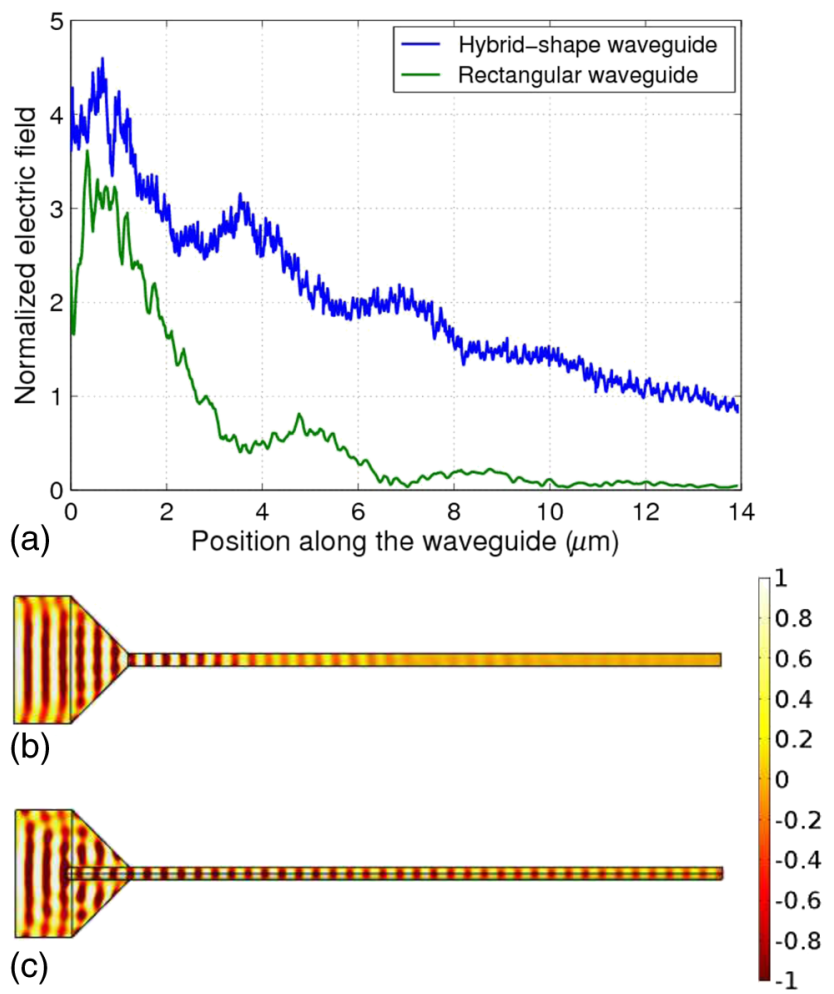

Fig. 6 (a) The normalized electric field distribution on the top surfaces of the rectangular (green) and modified stripe (blue) waveguides. (b) and (c) The distribution of a z-component of electric field on the rectangular and modified stripe waveguides, respectively. 
Figs. 6(b) and 6(c). Graphs show a marked improvement in the SPP propagation length in the case of the modified stripe waveguide in comparison with the rectangular waveguide crosssection.

Possible ways to fabricate such a waveguide shape involve either annealing ${ }^{27}$ or wet etching. ${ }^{28}$ In a first step, the base of a port can be deposited onto the silicon substrate. Then one of the proposed methods is performed, smoothing the corners of the rectangular waveguide. Both methods are compatible with CMOS technology.

\section{Conclusion}

In summary, the proposed design of modified stripe waveguide coupled with the plasmonic input port presents an opportunity for compact plasmonic integrated circuits. The mode analysis undertaken and changes in waveguide shape show the possibility of improving the propagation length of SPPs by a factor of up to 4.3 in comparison with a waveguide with a rectangular cross-section. The proposed structure is potentially CMOS compatible and very flexible, and could be adjusted to particular needs by using the design sequence described in this paper.

\section{Acknowledgments}

This research was supported under the Australian Research Council's Discovery Projects funding scheme (Project Number DP110100221).

\section{References}

1. M. T. Bohr and Y. A. El-Mansy, "Technology for advanced high-performance microprocessors," IEEE Trans. Electron Devices 45(3), 620-625 (1998).

2. M. F. Yanik et al., "All-optical transistor action with bistable switching in a photonic crystal cross-waveguide geometry," Opt. Lett. 28(24), 2506-2508 (2003).

3. S. John and M. Florescu, "Photonic bandgap materials: towards an all-optical micro-transistor," J. Opt. A: Pure Appl. Opt. 3(6), S103 (2001).

4. Y. Zhang, Y. Zhang, and B. Li, "Optical switches and logic gates based on self-collimated beams in two-dimensional photonic crystals," Opt. Express 15(15), 9287-9292 (2007).

5. J. Bai et al., "Photonic NOT and NOR gates based on a single compact photonic crystal ring resonator," Appl. Opt. 48(36), 6923-6927 (2009).

6. M. Born and E. Wolf, Principles of Optics: Electromagnetic Theory of Propagation, Interference and Diffraction of Light, Cambridge University Press, Cambridge, UK (1999).

7. J. J. Cadusch, T. D. James, and A. Roberts, "Experimental demonstration of a wave plate utilizing localized plasmonic resonances in nanoapertures," Opt. Express 21(23), 28450 28455 (2013).

8. J. J. Cadusch et al., "A chiral plasmonic metasurface circular polarization filter," IEEE Photonics Technol. Lett. 26(23), 2357-2360 (2014).

9. H. Wei et al., "Quantum dot-based local field imaging reveals plasmon-based interferometric logic in silver nanowire networks," Nano Lett. 11(2), 471-475 (2011).

10. L. Novotny and N. Van Hulst, "Antennas for light," Nat. Photonics 5(2), 83-90 (2011).

11. S. K. Earl et al., "Tunable optical antennas enabled by the phase transition in vanadium dioxide," Opt. Express 21(22), 27503-27508 (2013).

12. S. K. Earl et al., "Vanadium dioxide thickness effects on tunable optical antennas," Proc. SPIE 8923, 89232S (2013).

13. P. Neutens et al., "Electrical detection of confined gap plasmons in metal-insulator-metal waveguides," Nat. Photonics 3(5), 283-286 (2009).

14. J. Bodesheim and A. Otto, "On the quantitative measurement of the roughness spectrum of silver films," Surf. Sci. 45(2), 441-456 (1974).

15. B. Lamprecht et al., "Surface plasmon propagation in microscale metal stripes," Appl. Phys. Lett. 79(1), 51-53 (2001). 
16. E. Devaux et al., "Launching and decoupling surface plasmons via micro-gratings," Appl. Phys. Lett. 83(24), 4936-4938 (2003).

17. B. Hecht et al., "Local excitation, scattering, and interference of surface plasmons," Phys. Rev. Lett. 77(9), 1889 (1996).

18. A. Andryieuski and A. V. Lavrinenko, "Nanocouplers for infrared and visible light," Adv. OptoElectron. 2012, 839747 (2012).

19. D. K. Gramotnev and S. I. Bozhevolnyi, "Plasmonics beyond the diffraction limit," Nat. Photonics 4(2), 83-91 (2010).

20. J. H. Lau, Flip Chip Technologies, McGraw-Hill Professional, New York (1996).

21. P. B. Johnson and R.-W. Christy, "Optical constants of the noble metals," Phys. Rev. B 6(12), 4370 (1972).

22. G. Vuye et al., "Temperature dependence of the dielectric function of silicon using in situ spectroscopic ellipsometry," Thin Solid Films 233(1-2), 166-170 (1993).

23. I. S. Maksymov and Y. S. Kivshar, "Broadband light coupling to dielectric slot waveguides with tapered plasmonic nanoantennas," Opt. Lett. 38(22), 4853-4856 (2013).

24. J. Lu et al., "Numerical optimization of a grating coupler for the efficient excitation of surface plasmons at an Ag-SiO 2 interface," J. Opt. Soc. Am. B 24(9), 2268-2272 (2007).

25. S. A. Maier, Plasmonics: Fundamentals and Applications, Springer Science \& Business Media, Bath, UK (2007).

26. P. Berini, "Plasmon-polariton waves guided by thin lossy metal films of finite width: bound modes of symmetric structures," Phys. Rev. B 61(15), 10484 (2000).

27. W. Xiong et al., "Improvement of FinFET electrical characteristics by hydrogen annealing," IEEE Electron Device Lett. 25(8), 541-543 (2004).

28. R. Böhme et al. "Topography and roughness evolution of microstructured surfaces at laserinduced backside wet etching," Appl. Phys. A 80(2), 433-438 (2005).

Evgeniy Panchenko obtained his BSc and MEng (both with distinction) degrees in electronics engineering from Bauman Moscow State Technical University in 2009 and 2011, respectively. His current research interests include plasmonics, optical interconnects, optoelectronic systems, microelectronics, and optical detectors.

Timothy D. James obtained his BEng and $\mathrm{PhD}$ degrees in electronics engineering from the University of Western Australia in 2003 and 2009, respectively. He has been a research fellow at the University of St. Andrews (2009 through 2011) and the University of Melbourne (since 2011) as well as a technology fellow at the Melbourne Centre for Nanofabrication (2011 through 2015). His current interests involve the developing and fabrication of new types of optical metamaterials, MEMS, and imaging technologies.

Ann Roberts obtained her BSc (with first class honors and university medal) and $\mathrm{PhD}$ degrees in physics from the University of Sydney in Australia. She accepted an academic position in the School of Physics at the University of Melbourne, where she is now a professor. She has diverse research interests in physical optics and photonics. In particular, she has made significant advances in the computational and experimental study of plasmonic devices, metamaterials, and nanoscale antennas. 